\title{
Disposable sheath that facilitates endoscopic Raman spectroscopy
}

\author{
Wenbo Wang \\ Michael Short \\ Isabella T. Tai \\ Haishan Zeng
}

\section{SPIE.}




\title{
Disposable sheath that facilitates endoscopic Raman spectroscopy
}

\author{
Wenbo Wang, ${ }^{\text {a,b }}$ Michael Short, ${ }^{a}$ Isabella T. Tai,, ${ }^{c, d}$ and Haishan Zeng ${ }^{a, *}$ \\ ${ }^{\mathrm{a} B C}$ Cancer Agency Research Centre, Imaging Unit-Integrative Oncology Department, 675 West 10th Avenue, Vancouver, British Columbia, \\ V5Z 1L3, Canada \\ bUniversity of British Columbia, Faculty of Applied Science, Department of Biomedical Engineering, KAIS 5500, 2332 Main Mall, \\ Vancouver, British Columbia, V6T1Z4, Canada \\ ${ }^{\circ}$ BC Cancer Agency, Michael Smith Genome Sciences Centre, 675 West 10th Avenue, Vancouver, British Columbia, V5Z 1L3, Canada \\ dUniversity of British Columbia, Faculty of Medicine, Division of Gastroenterology, 2775 Laurel Street, Vancouver, British Columbia, V5Z 1M9, \\ Canada
}

\begin{abstract}
In vivo endoscopic Raman spectroscopy of human tissue using a fiber optic probe has been previously demonstrated. However, there remain several technical challenges, such as a robust control over the laser radiation dose and measurement repeatability during endoscopy. A decrease in the signal to noise was also observed due to aging of Raman probe after repeated cycles of harsh reprocessing procedures. To address these issues, we designed and tested a disposable, biocompatible, and sterile sheath for use with a fiber optic endoscopic Raman probe. The sheath effectively controls contamination of Raman probes between procedures, greatly reduces turnaround time, and slows down the aging of the Raman probes. A small optical window fitted at the sheath cap maintained the measurement distance between Raman probe end and tissue surface. To ensure that the sheath caused a minimal amount of fluorescence and Raman interference, the optical properties of materials for the sheath, optical window, and bonding agent were studied. The easy-to-use sheath can be manufactured at a moderate cost. The sheath strictly enforced a maximum permissible exposure standard of the tissue by the laser and reduced the spectral variability by 1.5 to 8.5 times within the spectral measurement range. ( 2016 Society of Photo-Optical Instrumentation Engineers (SPIE) [DOI: 10.1117/1.JBO.21.2.025001]
\end{abstract}

Keywords: Raman spectroscopy; endoscopy; disposable sheath; cross-contamination control; maximum permissible exposure; spectral variability.

Paper 150671TNR received Oct. 8, 2015; accepted for publication Dec. 22, 2015; published online Feb. 1, 2016.

\section{Introduction}

Colorectal cancer (CRC) is the second leading cause of cancerrelated deaths according to the latest statistics. ${ }^{1}$ The $\mathrm{CRC}$ incidence rates have actually declined both over the long-term and in very recent years. This suggests that both cancer screening programs and the increased uptake of screening techniques, mainly colonoscopy, to remove precursor lesions have been effective in reducing CRC incidence. ${ }^{2,3}$ Expanded screening programs would likely see further reductions in the CRC mortality rate.

Colonoscopy remains the gold standard for CRC diagnosis and offers therapeutic treatment (polypectomy) in the same session. ${ }^{4}$ However, a colonoscopy and the reprocessing of a colonoscope demand a significant amount of resources. An elevated demand for colonoscopy exams could greatly burden limited healthcare resources, making it impractical for a populationbased CRC screening program. ${ }^{5}$ Despite widely adopted device reprocessing and infection control guidelines, the time-consuming reprocessing procedures for endoscopes and the potential risk of cross-infection remain some of the major complications toward efficient gastrointestinal tract (GI) cancer screening. ${ }^{6}$ An innovative way to significantly decrease the turnaround time (setup time plus procedure time plus reprocessing time) and maintain a low probability of cross-infection is the use of a disposable sheath on endoscopic devices. ${ }^{7-11}$ Comparing the efficiency and productivity of disposable sheathed endoscopic devices with conventional equipment, there was an almost 10 -fold reduction in turnaround time. ${ }^{9}$ It was also clinically verified that the use of disposable sterile endoscopic sheaths could replace high-level disinfection in reprocessing. ${ }^{10}$ Combined with enzymatic detergent cleaning and disinfection using $70 \%$ ethanol, endoscopic instruments with disposable sheaths could be made ready for patient exams quickly and provide reliable protection against bacterial pathogen contamination. In addition, little difference was found in procedure time, imaging quality, and patient comfort between sheathed and conventional devices in randomized control trials. ${ }^{5,11}$ For a population-based CRC screening study, a disposable sterile endoscopic sheath was shown to be a practical tool and should be highly useful in decentralized screening in the future..$^{6,12}$

Another promising avenue of research for reducing CRC mortality rate is the use of adjunct devices in colonoscopy. The advent of real-time Raman spectroscopy technology and endoscopic probes has greatly facilitated in vivo Raman spectral acquisition. The oncological application of Raman spectroscopy has gradually gained traction in recent years. ${ }^{13}$ Previous research showed that near-infrared (NIR) Raman spectroscopy was capable of differentiating adenomas from hyperplastic polyps or normal colonic tissues in vivo. ${ }^{14,15}$ Raman fiber optic probes are designed to be passed down the narrow instrument channel of a standard GI endoscope to be able to take in vivo Raman 
spectra of colonic lesions. The construction of such Raman probes was quite challenging, requiring a probe diameter of $\sim 2$ to $3 \mathrm{~mm}$ (30 to 40 optic fibers) and length of 1.8 to $3.0 \mathrm{~m}$ with a high degree of flexibility and tensile strength. Meanwhile, there exists no standard reprocessing protocol for the Raman probes. To routinely clean and disinfect the Raman probes with endoscopes at the hospital reprocessing unit was impractical partially because of the high risk of damaging the expensive and delicate Raman probe. The harsh reprocessing protocol also speeds up the aging of the Raman probe, deteriorating its performance [decreasing signal-to-noise ratio (SNR)]. In addition to the device reprocessing requirement, measuring Raman spectra during endoscopic procedures needed a consistent sampling geometry to minimize spectral variability. Prior research efforts maintain the distance and tilting angles between the Raman probe tip and the colonic lesion surface sites solely based on manual control by the clinician with visual feedback from the real-time endoscopic video imaging. When spectral data were processed later, it was found that data consistency was compromised because the sampling geometry changed during measurement. The consequence could be significant because it decreases the predictive accuracy of the Raman measurement and may delay the procedure because of the need for repeated measurements. Therefore, it is crucial that some mechanisms be put in place to ensure the repeatability of clinical Raman measurement. The other consideration is patient safety when lesions are exposed to laser radiation. Although there are no official guidelines on the maximum permissible exposure (MPE) that colon tissue can be exposed to, the American National Standards Institute (ANSI) guidelines have set out MPE values for the human epidermis. Endoscopic Raman research studies have generally adopted the practice of not exceeding these published MPE values, but this is more challenging to do where the distance between probe and the tissue surface cannot be reliably controlled.

In this paper, we describe a disposable sterile endoscopic sheath to be used with a Raman probe and is compatible with a standard colonoscope instrument channel. The sheath allows the Raman probe to maintain a fixed geometry (both distance and angle) with tissue surface, thus leading to reduced spectral variability, and enforcing the MPE value of laser radiation. Considering the inherent weak Raman signals of live tissues, the optical properties of the sheath have to cause minimal interference to the spectra of colonic lesions. Therefore, this paper reports on issues ranging from material selection to measurement tests of live human tissues.

\section{Materials and Methods}

\subsection{Instrument Setup}

The design of the real-time Raman spectrometer was adapted from an instrument setup for lung cancer in vivo measurement and was described previously. ${ }^{14,16}$ In brief, excitation from a 785-nm 300-mW stabilized diode laser (model BRM-785, B\&W Tek, Newark, Delaware) was coupled into a bifurcated endoscopic Raman probe. Collected emissions were analyzed with an $f / 2.2$ spectrograph (model Holospec, Kaiser Optical Systems, Ann Arbor, Michigan) working from 1531 to $3416 \mathrm{~cm}^{-1}$. The power of the excitation light at the sample was around $140 \mathrm{~mW}$. An NIR optimized, back-illuminated, deep depletion charge coupled device $(\mathrm{CCD})$ with $400 \times 1340$ pixels $(20 \times 20 \mu$ m per pixel) was used for detection (model Spec-10: 400BR/LN, Princeton Instruments, Trenton, New Jersey). The 3-m-long Raman probe (2.3 $\mathrm{mm}$ in diameter) was enclosed in a stainless steel monocoil to give it increased tensile strength while maintaining a high degree of flexibility. To protect the probe from biological contaminants, it was covered with a medical-grade heat shrink tubing (Iridium, Cobalt Polymers, Cloverdale, California). The central $200-\mu$ m-diameter lone delivery fiber was surrounded by 34 collection fibers (100 $\mu \mathrm{m}$ in diameter). Another specially designed optic fiber bundle was employed to couple collected light to the spectrograph. ${ }^{17}$ Wavelength calibration was done with mercury argon $(\mathrm{Hg} / \mathrm{Ar})$ and xenon $(\mathrm{Xe})$ calibration lamps (Ocean Optics, Dunedin, Florida) and spectral resolution was estimated to be $\approx 6 \mathrm{~cm}^{-1}$ at $1013 \mathrm{~nm}$ (corresponding to a Raman shift of $2867 \mathrm{~cm}^{-1}$ with $785-\mathrm{nm}$ excitation).

\subsection{Sheath Design}

A low-cost sheath material needs to be biocompatible, mechanically able to meet the required needs, and contribute minimal interfering fluorescence or Raman emissions. The material chosen was polyvinylidene difluoride (PVDF), a transparent, slightly flexible material with high tensile strength that can be manufactured into tubes of different sizes. PVDF is biocompatible and well suited for biomedical applications. It does have some Raman features, but since the material is not directly placed in the excitation and collection optical paths (not used as the window), it was not expected to have a significant effect on the tissue material Raman. Specifically in the fingerprint region, and below $1000 \mathrm{~cm}^{-1}$, PVDF has Raman emission mainly dominated by two peaks ${ }^{18}$ at 795 and $839 \mathrm{~cm}^{-1}$. Between 1000 and $1800 \mathrm{~cm}^{-1}$, the PVDF generates an intense Raman band at $\sim 1433 \mathrm{~cm}^{-1}$, originating from the $\mathrm{CH}_{2}$ bending mode, atop of a flat featureless spectral background. In the high wavenumber region $\left(\sim 2400\right.$ to $\left.3800 \mathrm{~cm}^{-1}\right)$, a relatively strong Raman peak around $3000 \mathrm{~cm}^{-1}$ exists. ${ }^{19}$

A PVDF tube with a $0.15-\mathrm{mm}$ wall thickness has a good balance between flexibility and rigidity as well as an intermediate value in terms of coefficient of friction when compared to other available materials. To maintain a fixed distance between the Raman probe tip and the tissue surface, an optical spacer (window) was designed and attached to one end of the PVDF tube using medical-grade epoxy (Epotek 301, Epoxy Technology Inc., Billerica, Massachusetts). The length of the spacer was designed according to the illumination spot size originally calculated in previous research, and taking into account the refractive index of the material. Both entrance and exiting surfaces of the optical spacer had an antireflective coating on them. The disposable sheath and Raman probe assembly is shown in Fig. 1.

\subsection{Data Handling and Software}

A commercial software product, WinSpec/32 (Version 2.5.20.2, Princeton Instruments), was used to operate the spectrometer and acquire Raman spectra. The CCD pixel rows were binned orthogonally to the wavenumber axis to enhance the SNR of the spectra. ${ }^{17}$ A 13-point third-order Savitzky-Golay smoothing filter using built-in functions in LabVIEW (National Instruments, Austin, Texas) was applied to smooth the Raman spectra. ${ }^{20}$ The CCD dark noise background was subtracted from acquired spectra. ${ }^{21}$ Fluorescence background was also removed using a fifth-order polynomial fitting algorithm with a window size of 5 and a stopping criteria of $<5 \%$ between the standard deviation of fitting residuals for two iterations. $^{22}$ 


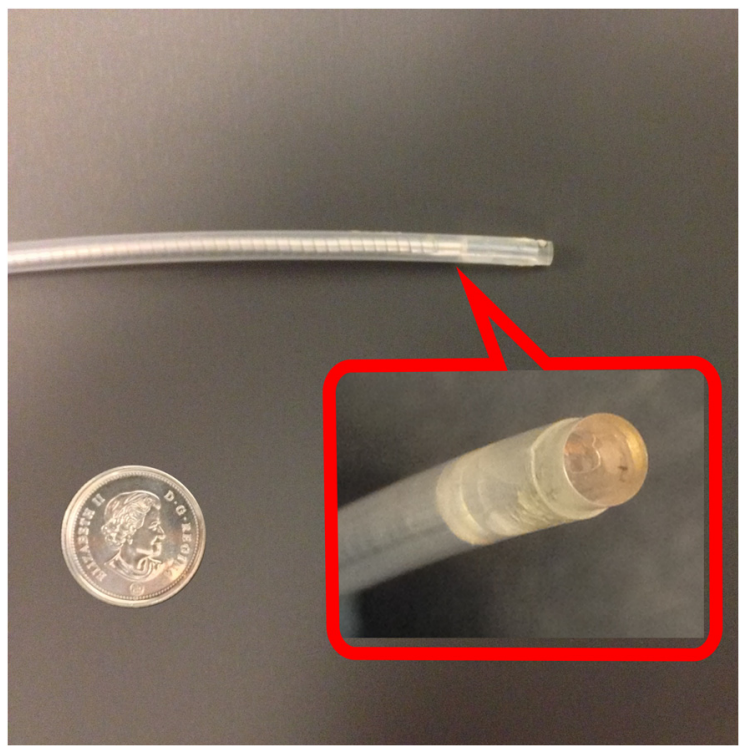

Fig. 1 The Raman probe and disposable sheath assembly with a Canadian dime (diameter: $18.03 \mathrm{~mm}$ ) for scale. Inset: zoomed-in view of the optical spacer at the front end.

\section{Results and Discussion}

\subsection{Spectral Property of Spacer Glass}

Because both excitation and emission light transmit directly through the optical window at the sheath tip, the window material must contribute only a minimal amount of spectral interference to the Raman measurement. In addition, the selected optical material needs to be biocompatible and economically affordable. Quartz and fused silica windows meet the required biocompatibility and cost requirements. To test spectral interference levels incurred by these materials under laser radiation, in vivo Raman spectra of palm skin from a volunteer were collected with either optical-grade quartz or fused silica windows ( $1 \mathrm{~cm}$ in thickness) placed in between the Raman optic fiber probe and the skin surface (Fig. 2). The spectra were first normalized to a common peak at $1657 \mathrm{~cm}^{-1}$ and then shifted vertically for clarity. As is evident in Fig. 2, the Raman spectrum measured through quartz window was accompanied by strong fluorescence between 1690 and $2500 \mathrm{~cm}^{-1}$, which overwhelmed the Raman peak at $1657 \mathrm{~cm}^{-1}$. The spectral difference in Fig. 2(b) shows the strong fluorescence (1690 and $2500 \mathrm{~cm}^{-1}$ ) for the quartz window and its reduced transmission that weakens the skin Raman peak intensity between 2800 and $3000 \mathrm{~cm}^{-1}$. The difference between normalized Raman spectra acquired with the probe alone and with the fused silica window is close to zero across the entire spectral region [Fig. 2(b)]. Therefore, the fused silica window in the optical path did not interfere with Raman measurement significantly and Raman peaks pertaining to skin tissues could be preserved with a minimum amount of distortion. Therefore, fused silica is considered the preferred material for constructing the probe sheath tip in this application.

\subsection{Spectral Property of Epoxy and Polyvinylidene Difluoride Sheath Wall}

When the Raman probe is sheathed, excitation laser light reflected from either the fused silica window surfaces or the tissue surface could return to the sheath and generate unwanted background signals. Although this interference is expected to be minimal, it was important to confirm this by measuring the Raman spectra of the epoxy and the sheath material. To test the epoxy, a 1-mm-thick epoxy resin was deposited onto a piece of anodized aluminum foil and allowed to dry for $48 \mathrm{~h}$. To test the sheath, multiple small sections of the wall were cut from a piece of PVDF tubing and stacked to a thickness of $0.6 \mathrm{~mm}$ on top of a piece of anodized aluminum foil. The first experiment on these samples used the same measurement conditions as for in vivo human tissue with the following measurement parameters: excitation power $140 \mathrm{~mW}$, integration time $1 \mathrm{~s}$, and probe to sample distance $7.5 \mathrm{~mm}$. The second

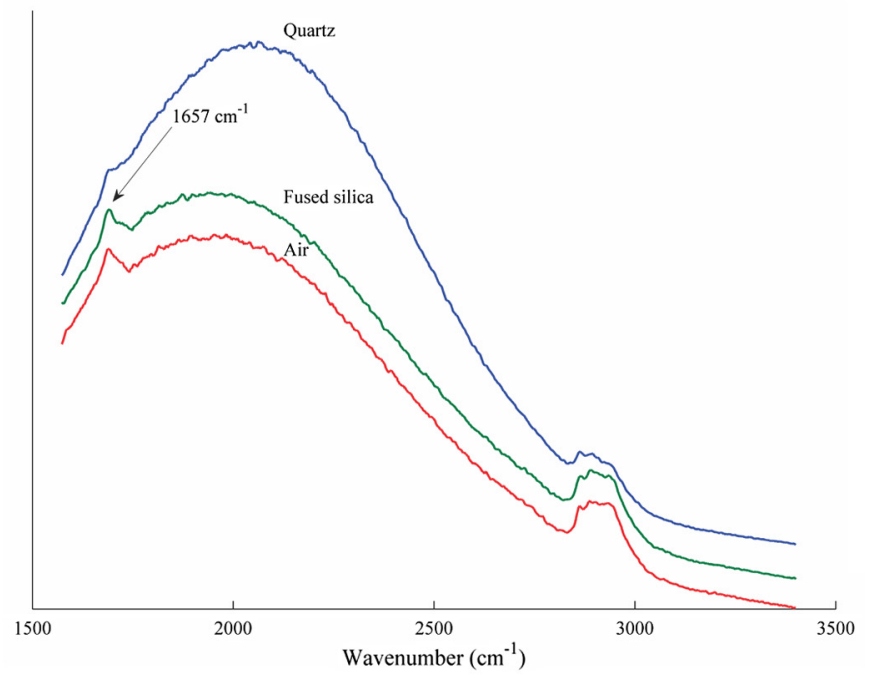

(a)

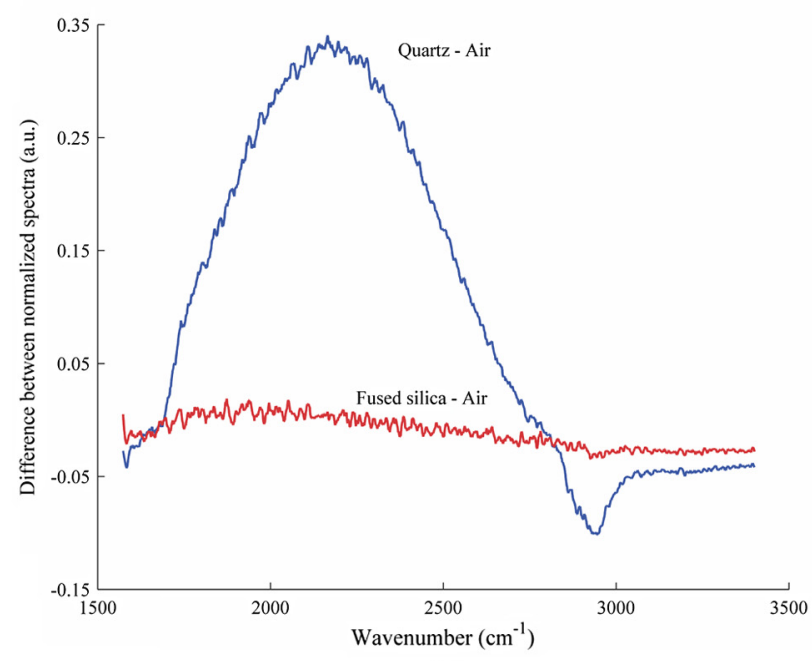

(b)

Fig. 2 In vivo Raman skin spectra collected with quartz, fused silica, or air between palm skin surface and optic fiber probe: (a) raw Raman spectra and (b) difference spectra between normalized Raman spectra acquired with and without optical window. 
experiment on these samples was designed to clearly identify unique Raman features for epoxy and PVDF by increasing the amount of collected light. To achieve this, the sampling distance was reduced to $0.5 \mathrm{~mm}$ and integration time was set to $10 \mathrm{~s}$ with the same excitation power as in the in vivo measurements. The spectra of anodized aluminum foil was also acquired as the background and subtracted from the Raman spectra for PVDF and epoxy. Figure 3(a) shows the raw Raman spectra for skin from a volunteer, the epoxy, and the PVDF tubing stack obtained in the first experiment. Apparently, skin generated the highest level of fluorescence background compared to sheath materials. The sharp peak at $1657 \mathrm{~cm}^{-1}$ and Raman band between 2800 and $2940 \mathrm{~cm}^{-1}$, which belong to skin, could be easily identified. There was a small peak around $3000 \mathrm{~cm}^{-1}$ for PVDF and a peak at $1613 \mathrm{~cm}^{-1}$ for epoxy resin. The common peak around $1780 \mathrm{~cm}^{-1}$ in epoxy and PVDF spectra was believed to belong to the optic fiber. Figure 3(b) shows the results obtained in the second experiment. In this case, the Raman peaks for epoxy and PVDF are greatly enhanced with optimized sampling geometry and 10 times integration time. The epoxy showed a sharp spectra peak at $1613 \mathrm{~cm}^{-1}$ and a relatively broad peak from 2850 to $2950 \mathrm{~cm}^{-1}$. The latter overlaps with the Raman features of skin, as does the Raman peak around $3000 \mathrm{~cm}^{-1}$ from the PVDF, which is in agreement with previous reports. ${ }^{19}$ Although easily distinguishable, it should be noted that the Raman spectra for both PVDF and epoxy as shown in Fig. 3(a) were obtained with full excitation power and with a geometry that maximized the collection of any emission from these samples. Under actual tissue measurement conditions [Fig. 3(a), top curve], both PVDF and epoxy were only exposed to laser light specularly reflected from tissue and spacer window surfaces, which accounted for only 1 to $2 \%$ of the total excitation power, and any excitation of these materials would occur outside of the aperture for direct collection by the Raman probe fibers. Furthermore, both the epoxy and PVDF were also at least eight times thinner in the assembled sheath, which would also lead to a much lower background signal from these materials. Thus, despite their clear Raman features [Fig. 3(a), bottom and middle curves], the spectral contribution from PVDF and epoxy to live tissue measurement could be considered minimal.

\subsection{Signal Collection Efficiency}

It is of interest to find out how the signal collection efficiency varies with sampling distance between the tissue surface and Raman probe end. A Tylenol tablet (acetaminophen) was placed underneath the Raman probe and moved vertically by a micrometer sampling stage. The total excitation power from the laser was kept constant. The distance was gradually increased from 0.1 to $10 \mathrm{~mm}$. Three major Raman peaks at 1648, 2931, and $3064 \mathrm{~cm}^{-1}$ were selected [Fig. 4(a)]. The intensity versus sampling distance of the three Raman peaks after fluorescence background removal is shown in Fig. 4(b).

As is seen in Fig. 4, the collected Raman peak signal reaches its maximum point when the sampling distance is $\sim 0.5 \mathrm{~mm}$. Thus, the sampling distance of $0.5 \mathrm{~mm}$ suggested that the collection is most efficient for such Raman probe design. However, this does not take into account the multiple scattering events that occur in translucent tissue samples, which would result in a collection efficiency maximum at a greater probe distance. One drawback with short probe to tissue distances is the illumination spot is only $\sim 0.04 \mathrm{~mm}^{2}$, which is too small to be able to acquire representative Raman spectra of the tumor tissue volume. In addition, the excitation power would have to be reduced drastically to comply with the MPE limit for skin set out in the ANSI guidelines. With an illumination spot size of diameter $3.5 \mathrm{~mm}$, a spectrum is obtained from a tissue volume that is more representative of the bulk tissue and a much higher excitation power can be used to compensate for the greater probe to tissue distance. Therefore, the sampling distance was optimally set at $7.95 \mathrm{~mm}$ to produce a 3.5 -mm-diameter excitation spot, and an

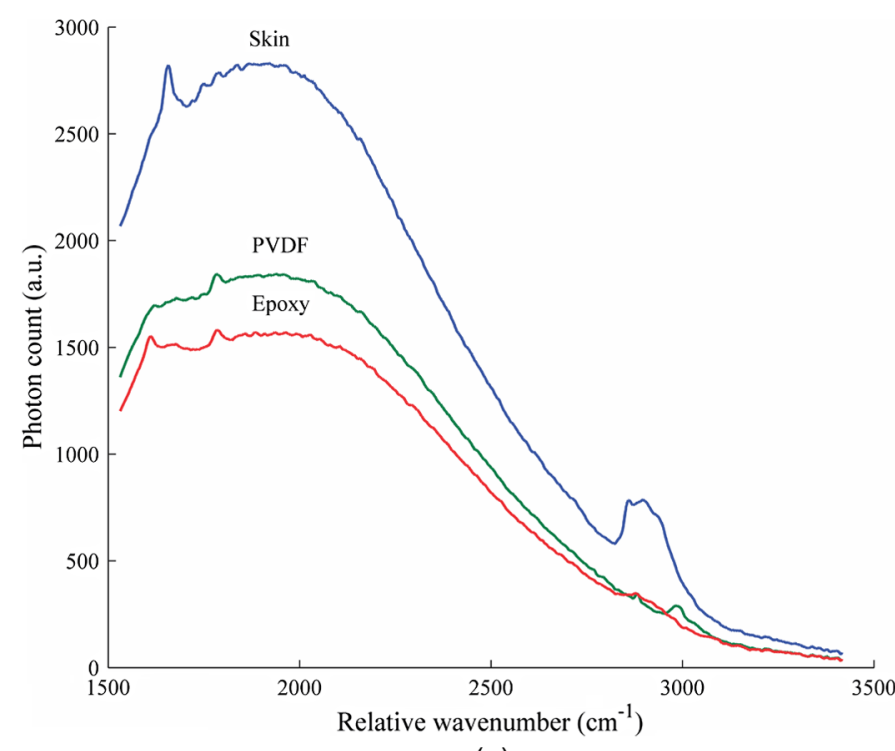

(a)

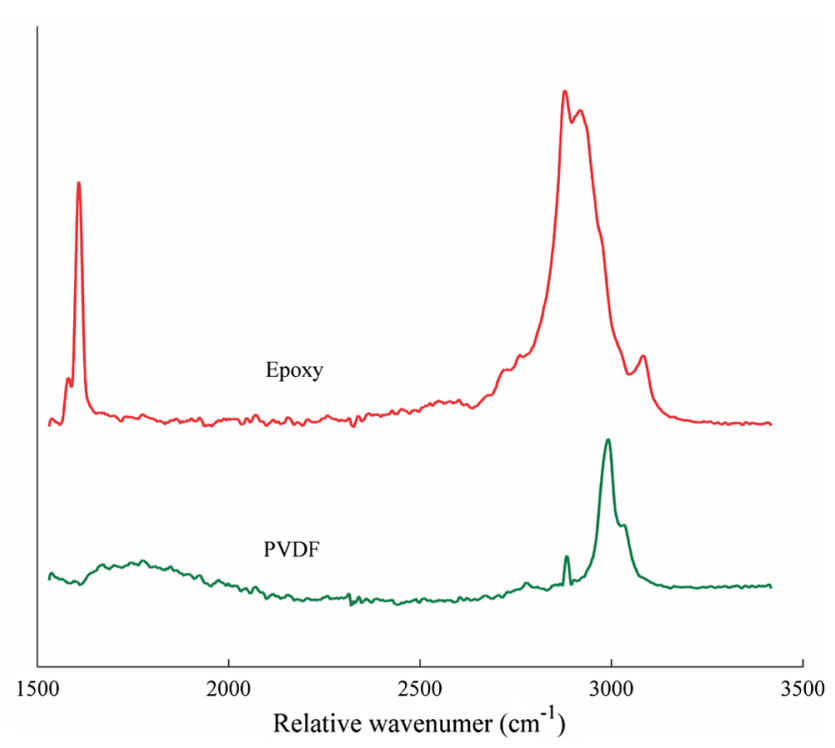

(b)

Fig. 3 Raman spectra of PVDF (sheath wall) and epoxy resin deposited on aluminum foil: (a) raw Raman spectra for palm skin, epoxy resin, and PVDF polymer as measured in experiment 1 and (b) Raman spectra of epoxy resin and PVDF on top of anodized aluminum foil in experiment 2 . The spectrum of anodized aluminum foil was subtracted from Raman spectra of PVDF and epoxy. Spectra were shifted on the intensity scale for clarity. 


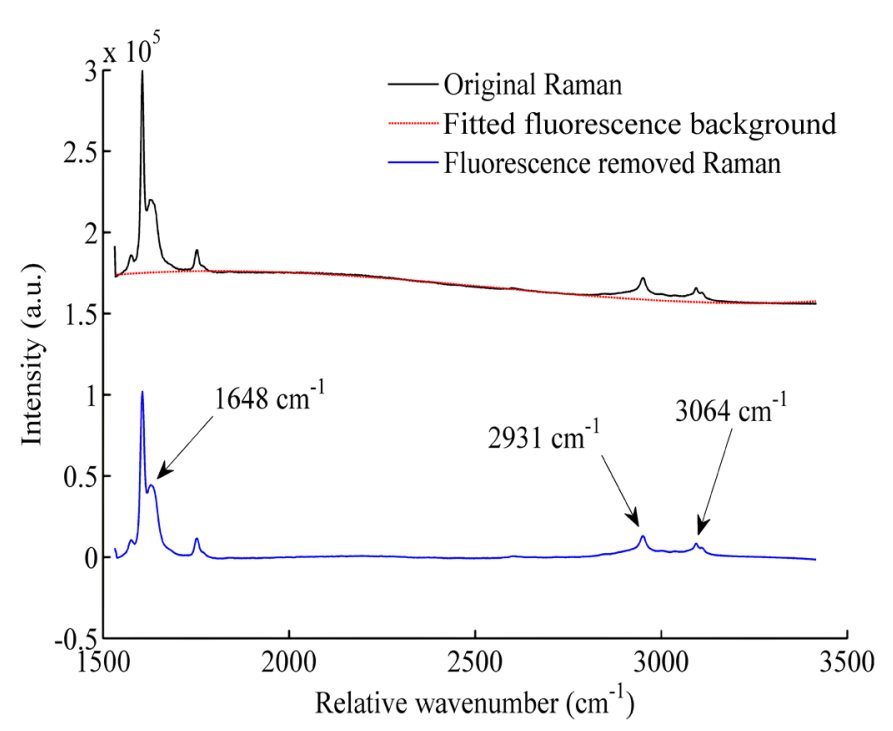

(a)

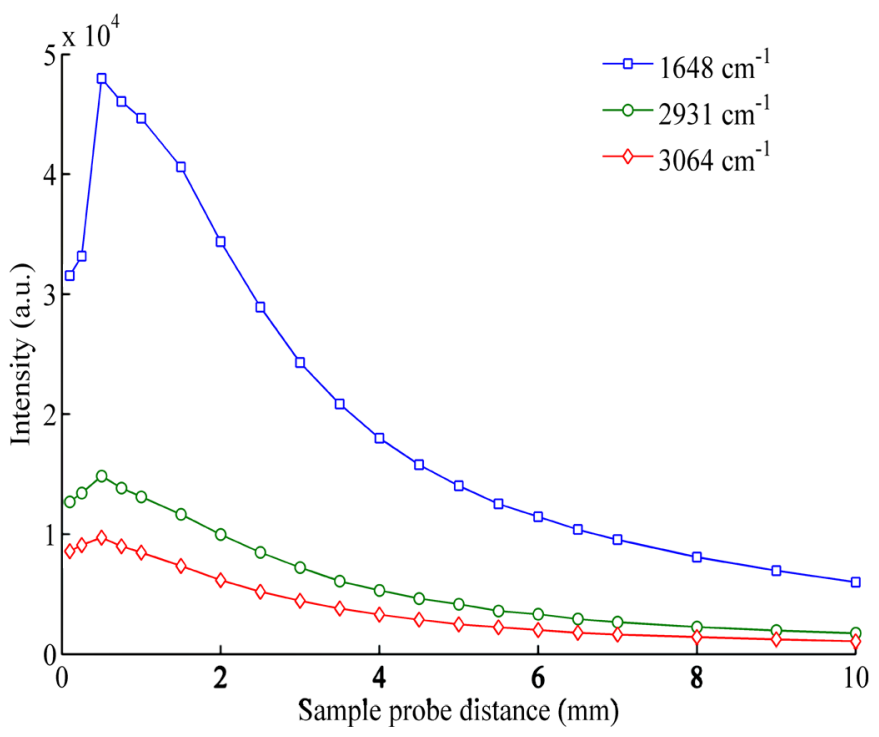

(b)

Fig. 4 Variation in collected spectral intensity of Raman peaks: (a) Raman spectra of acetaminophen and (b) variation in intensity of peaks at 1648,2931 , and $3064 \mathrm{~cm}^{-1}$ with changes in distance between Raman probe and the Tylenol tablet.

excitation power of $140 \mathrm{~mW}$ used here complied with the MPE limit for human epidermis $(1.628 \mathrm{~J} / \mathrm{cm}$ with $785 \mathrm{~nm}$ excitation and an integration time of $1 \mathrm{~s}$ ). Without the sheath, any slight deviation from the sampling distance toward the tissue site will lead to patients being exposed to a higher dose of laser radiation. Technically, it was challenging to maintain a proper sampling distance during an endoscopic exam where the physician could only make a best judgment about sampling distance based on white light imaging. Therefore, the presence of the Raman sheath ensures that the MPE value of the laser radiation on human epidermis is not exceeded.

\subsection{Spectra Repeatability}

The Raman peak intensity tends to vary greatly when the sampling distance changes [Fig. 4(b)]. The peak intensity variation with changing sampling distance unnecessarily complicates endoscopic Raman measurement by introducing measurement errors. Such variation was partially caused by collection efficiency as well as by the change in excited tumor volume. In

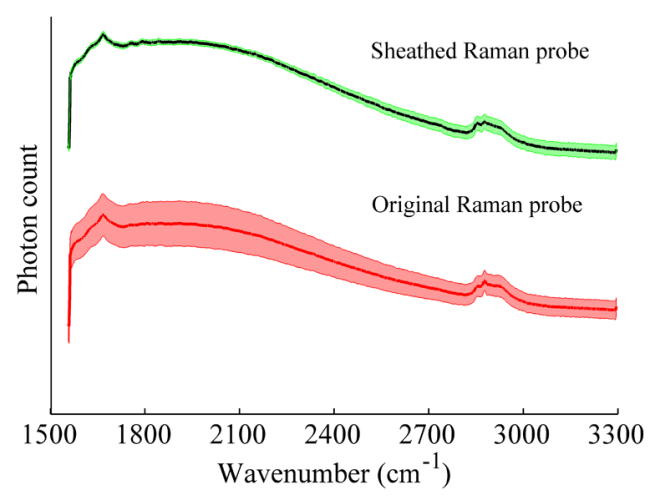

Fig. 5 The mean and standard deviation of measured Raman spectra of palm skin using Raman probe with and without the designed disposable sheath. Spectra were intentionally shifted on the intensity scale for clarity. the endoscope, the only method to adjust the sampling distance was to pull/push the Raman fiber optic bundle through the instrument channel. Therefore, the lack of precision in controlling the sampling distance was anticipated under most operating circumstances. In Fig. 5, the variation in acquired Raman spectra with and without the disposable Raman sheath is demonstrated. Each type of spectra measurement was repeated 20 times by the same operator on the same skin site (human hand palm). The unsheathed Raman probe was held at a distance between 6 and $8 \mathrm{~mm}$ away from the skin surface, mimicking the measurement conditions during endoscopic measurement.

Figure 5 shows that the spectral variation due to varying sampling distance could be effectively reduced by using the disposable sheath during measurement. On average, the coefficient of variation could be reduced by 1.5 to 8.5 times depending on the spectral range by simply using the sheath.

\section{Conclusions}

Colonoscopy is effective in preventing CRC; thus, the demand for colonoscopy exams in CRC screening programs is expected to continue to grow. To be able to screen the eligible population without straining the limited colonoscopy resources, the exam turnaround time must be drastically reduced. Real-time endoscopic Raman spectroscopy offers in vivo and subjective diagnosis of suspicious colonic lesions based on molecular signatures specific to cancer growth. Thus, using Raman spectroscopy as an adjunct diagnosis technique to conventional colonoscopy can potentially obviate the need for some unnecessary polypectomy and histopathology work. However, stringent reprocessing requirements limit its utility in population screening. In this paper, a disposable sheath specially designed for endoscopic Raman spectroscopy was introduced. The sheath not only provided a viable option to drastically reduce reprocessing time, but also ensured that the set MPE of the tissue was not exceeded. The construction materials for the disposable sheath were studied to clearly identify their potential contributions, which were minimal, to tissue Raman measurement. The 
repeatability of spectral data was also greatly improved by 1.5 to 8.5 times with sheathed Raman probe.

\section{Acknowledgments}

Financial support provided by the Canadian Institutes of Health Research is gratefully acknowledged. The authors would like to thank Jianhua Zhao and Hanna Pawluk for helpful comments to improve the manuscript. W.W. gratefully acknowledges the postdoctoral fellowship provided by the Engineer-in-Scrubs program at the Department of Biomedical Engineering, University of British Columbia.

\section{References}

1. R. L. Siegel, K. D. Miller, and A. Jemal, "Cancer statistics, 2015," CA Cancer J. Clin. 65(1), 5-29 (2015).

2. B. K. Edwards et al., "Annual report to the nation on the status of cancer, 1975-2006, featuring colorectal cancer trends and impact of interventions (risk factors, screening, and treatment) to reduce future rates," Cancer 116(3), 544-573 (2010).

3. R. L. Siegel, E. M. Ward, and A. Jemal, "Trends in colorectal cancer incidence rates in the United States by tumor location and stage, 19922008," Cancer Epidemiol. Biomarkers Prev. 21(3), 411-416 (2012).

4. T. M. Geiger and R. Ricciardi, "Screening options and recommendations for colorectal cancer," Clin. Colon Rectal Surg. 22(4), 209-217 (2009).

5. B. Mayinger et al., "Disposable-sheath, flexible gastroscope system versus standard gastroscopes: a prospective, randomized trial," Gastrointest. Endosc. 50(4), 461-467 (1999).

6. P. Jin et al., "Safety and efficacy of a novel disposable sheathed gastroscopic system in clinical practice," J. Gastroenterol. Hepatol. 29(4), 757-761 (2014).

7. R. I. Rothstein and B. Littenberg, "Disposable, sheathed, flexible sigmoidoscopy: a prospective, multicenter, randomized trial. The Disposable Endoscope Study Group," Gastrointest. Endosc. 41(6), 566-572 (1995).

8. P. C. Schroy, III, S. Wilson, and N. Afdhal, "Feasibility of high-volume screening sigmoidoscopy using a flexible fiberoptic endoscope and a disposable sheath system," Am. J. Gastroenterol. 91(7), 1331-1337 (1996).

9. T. C. Sardinha et al., "Efficiency and productivity of a sheathed fiberoptic sigmoidoscope compared with a conventional sigmoidoscope," Dis. Colon Rectum 40(10), 1248-1253 (1997).

10. C. J. Alvarado, A. G. Anderson, and D. G. Maki, "Microbiologic assessment of disposable sterile endoscopic sheaths to replace high-level disinfection in reprocessing: a prospective clinical trial with nasopharygoscopes," Am. J. Infect. Control 37(5), 408-413 (2009).

11. A. Krebs et al., "Evaluation of practice efficiency with a novel sheathed flexible cystoscope: a randomized controlled trial," Urology 70(5), 883887 (2007).

12. M. Bretthauer et al., "Use of a disposable sheath system for flexible sigmoidoscopy in decentralized colorectal cancer screening," Endoscopy 34(10), 814-818 (2002).

13. W. Wang et al., "Real-time in vivo cancer diagnosis using Raman spectroscopy,” J. Biophotonics 8(7), 527-545 (2015).
14. M. A. Short et al., "Using high frequency Raman spectra for colonic neoplasia detection," Opt. Express 21(4), 5025-5034 (2013).

15. A. Molckovsky et al., "Diagnostic potential of near-infrared Raman spectroscopy in the colon: differentiating adenomatous from hyperplastic polyps," Gastrointest. Endosc. 57(3), 396-402 (2003).

16. M. A. Short et al., "Development and preliminary results of an endoscopic Raman probe for potential in vivo diagnosis of lung cancers," Opt. Lett. 33(7), 711-713 (2008).

17. Z. W. Huang et al., "Rapid near-infrared Raman spectroscopy system for real-time in vivo skin measurements," Opt. Lett. 26(22), 1782-1784 (2001).

18. T. Boccaccio et al., "Characterization of PVDF membranes by vibrational spectroscopy," J. Membrane Sci. 210(2), 315-329 (2002).

19. Y. L. Ji et al., "Analysis of Raman and infrared spectra of poly (vinylidene fluoride) irradiated by $\mathrm{KrF}$ excimer laser," Spectrochim. Acta A 70(2), 297-300 (2008).

20. A. Savitzky and M. J. E. Golay, "Smoothing and differentiation of data by simplified least squares procedures," Anal. Chem. 36(8), 1627-1639 (1964).

21. J. H. Zhao et al., "Integrated real-time Raman system for clinical in vivo skin analysis," Skin Res. Technol. 14(4), 484-492 (2008).

22. J. Zhao et al., "Automated autofluorescence background subtraction algorithm for biomedical Raman spectroscopy," Appl. Spectrosc. 61(11), 1225-1232 (2007).

Wenbo Wang is a postdoctoral fellow at the BC Cancer Agency Research Centre and the Department of Biomedical Engineering, University of British Columbia. He holds BS and MS degrees in precision instruments from Tianjin University and $\mathrm{PhD}$ in biosystems engineering from University of Manitoba. His current research interests include applied spectroscopy instrumentation, laser scanning microscopy, and biophotonics. He is a member of SPIE.

Michael Short is a research associate with the Integrative Oncology Department (Imaging Unit), British Columbia Cancer Agency Research Centre. He obtained his BSc in physics from the University of Lancaster, United Kingdom, his MPhil from the University of Hong Kong, China, and his PhD from Simon Fraser University, Canada. He has published over 30 papers and filed three patents. He is a member of the SPIE. His research interests are thermoluminesence, optically stimulated luminescence, and biomedical photonics.

Isabella T. Tai is an associate professor with the Department of Gastroenterology, University of British Columbia, and a senior scientist with the Genome Sciences Centre at BC Cancer Agency. Her research focuses on gastrointestinal cancers using genomics and proteomics approaches. She holds 18 granted patents related to colorectal cancer diagnosis and therapy.

Haishan Zeng is a distinguished scientist with the Imaging UnitIntegrative Oncology Department at BCCRC and a professor of dermatology, pathology, and physics at the University of British Columbia. He obtained his $\mathrm{PhD}$ in biophysics from the University of British Columbia. His research focuses on developing optical imaging and spectroscopy techniques for early cancer detection. He has published over 140 refereed journal papers, 1 book, 15 book chapters, and has 21 granted patents. 